of T-cells into a regulatory phenotype. In a murine B16 xenograft model IL-2 expression significantly enhanced therapeutic effects of an $\mathrm{H} 1$ oncolytic influenza virus. Expressed within the background of H5 hemagglutinin, IL-2 did not lead to a significant enhancement of therapeutic efficacy. Interestingly, the empty influenza H5 subtype was significantly more potent in treating B16 xenograft tumors than the H1 subtype, regardless of IL-2 expression. In primary human PBMC models, the virus based on $\mathrm{H} 1$ hemagglutinin led to enhanced CD8 T-cell activation compared to H5. This effect was further enhanced by IL-2 expression, although all viruses led to significant activation. Surprisingly, viruses based on H1 hemagglutinin led to increased expression of the immune checkpoint PD-1. The virus based on H5 hemagglutinin did not lead to upregulation of PD-1, indicating a favorable balance between activation and exhaustion. Infection with the $\mathrm{H} 5$ based virus led to both enhanced apoptosis and immunogenic calreticulin exposure in human and murine melanoma cell lines compared to $\mathrm{H} 1$.

Conclusions IL-2 does not promote T-regs, when expressed in a viral background. $\mathrm{H} 1$ viruses induced $\mathrm{PD}-1$ more potently than $\mathrm{H} 5$ viruses. The choice of viral entry protein is more relevant for the therapeutic effect of the virus, than the expression of a T-cell stimulating cytokine such as IL-2. Efficacy of oncolytic viral treatment appears to depend more on viral growth than on virally expressed T-cell promoting cargo. Disclosure Information J. Kabiljo: None. I. Kuznetsova: None. J. Homola: None. S. Prodinger: None. J. Laengle: None. M. Sachet: None. A. Egorov: A. Employment (full or part-time); Modest; Vacthera Bio Tech GmbH. M. Bergmann: A. Employment (full or part-time); Modest; Vacthera Bio Tech GmbH.

\section{P09.05 IMMUNOGENICITY INDUCED BY THE ACADEMIC CHIMERIC ANTIGEN RECEPTOR CAR19 (ARI-0001) IN PATIENTS WITH CD19-POSITIVE RELAPSED/REFRACTORY B-CELL MALIGNANCIES RECRUITED INTO THE CART19- BE-01 CLINICAL TRIAL}

\begin{abstract}
1,2N Klein-González* ${ }^{*}$ 1,2EA González-Navarro, 1,2 A Bartoló-Ibars, ${ }^{3} \mathrm{~V}$ Ortiz-Maldonado, ${ }^{4} \mathrm{M}$ Torrebadell, $5,6 \mathrm{M}$ Castellà, ${ }^{1,2} \mathrm{D}$ Benítez, 1,2M Caballero-Baños, 1,2R Cabezón, 1,2 M Español, ${ }^{3} \mathrm{~T}$ Baumann, ${ }^{3} \mathrm{E}$ Giné, ${ }^{7,8} \mathrm{P}$ Castro, ${ }^{3,5,8}$ J Esteve, ${ }^{2,5,8}$ J Yagüe, ${ }^{4} \mathrm{~S}$ Rives, ${ }^{3,5,8} \AA_{\mathrm{A}}$ Álvaro Urbano-Ispizua, ${ }^{3,5}$ J Delgado, $1,2,5,8 \mathrm{M}$ Juan. ${ }^{1}$ Immunotherapy Unit, HSJD-Hospital Clínic Barcelona, Barcelona, Spain; ${ }^{2} / m m u n o l o g y, ~ C D B$, Hospital Clínic Barcelona, Barcelona, Spain; ${ }^{3}$ Hematology, ICMHO, Hospital Clínic Barcelona, Barcelona, Spain; ${ }^{4}$ Hematology, Hospital Sant Joan de Déu (HSJD), Barcelona, Spain; Institut d'Investigacions Biomèdiques August Pi i Sunyer - IDIBAPS, Barcelona, Spain; ${ }^{6}$ Banc de Sang i Teixits (BST), Barcelona, Spain; ${ }^{7}$ Medical Intensive Care Unit, Hospital Clínic Barcelona, Barcelona, Spain; ${ }^{8}$ University of Barcelona, Barcelona, Spain
\end{abstract}

\subsection{6/jitc-2020-ITOC7.105}

Background Chimeric Antigen Receptor (CAR)-T cells directed against CD19 have induced high rates of response in patients with relapsed/refractory (R/R) B-cell malignancies. Two CD19targeting constructs have been approved by the FDA and EMA (Yescarta ${ }^{\circledR}$, Kymriah ${ }^{\circledR}$ ) for B lymphoblastic leukemia (B-ALL) and aggressive lymphoma. Despite deep remissions, there are still major challenges and disparate data are reported about the immunogenicity induced by CART-cell therapy. On May/2017, the Spanish Agency of Medicines approved our first clinical trial (clinicaltrials.gov NCT03144583) with a fully academic CART-19.

Materials and Methods Eligibility criteria included R/R B-ALL (adult and pediatric), non-Hodgkin's lymphoma (NHL) and chronic lymphocytic leukemia(CLL) who failed standard therapy. The primary objective of the study was safety and secondary objectives were response rate and its duration. The humoral anti-CART response was assessed by a (cell-based) fluorescence assay to detect human anti-murine antibodies (HAMA) in patients sera. Assessment was conducted at different time points: 1) at baseline (pre-dose), 2) on day 14 after the administration of ARI-0001 cells, 3) on day 28, 4) on day 100, and 5) every 3 months thereafter.

Results Forty-seven patients (37 adults/10 pediatrics) received ARI-0001 cells. Thirty-eight patients had a diagnosis of $\mathrm{R} / \mathrm{R}$ B-ALL (28 adults and 10 children); all but 5 had relapsed after allogeneic hematopoietic stem cell transplant (HCT). Seven patients had a diagnosis of NHL, four of them $(57 \%)$ had relapsed after HCT, and 2 patients had a diagnosis of CLL (2). Median age was 27 years (3-68). After conditioning with fludarabine $(90 \mathrm{mg} / \mathrm{m} 2)$ and cyclophosphamide $(900$ $\mathrm{mg} / \mathrm{m} 2$ ), a total dose of $0.5-5 \times 10^{6}$ ARI-0001 cells $/ \mathrm{kg}$ was infused. Autologous T-cells from peripheral blood were expanded and transduced with a lentivirus to express a CAR with a single-chain variable fragment $(\mathrm{scFv})$ with anti-CD19 specificity, conjugated with the co-stimulatory regions $4-1 \mathrm{BB}$ and $\mathrm{CD} 3 \mathrm{z}$. The $\mathrm{scFv}$ was originated from a mouse monoclonal antibody A3B1. Twenty-five per cent of the patients tested positive for the presence of anti-CAR antibodies, all of them post-dose, in contrast to previous data reported on Kymriah $^{\circledR}$ with a significant presence of pre-dose anti-murine CAR19 antibody. Of these 12 patients, 8 patients presented with a weak, and 4 patients with a strong presence of HAMA. The last 4 patients had lost the effectiveness of the CART- therapy at that time point, reflected by simultaneous B-cell recovery in the periphery. Moreover, three of them received a second dose of CART-19, which did not revert the relapse.

Conclusions To conclude, these data suggest the importance of the immunogenicity induced by CART-cell therapies. Immune monitoring should include the assessment of humoral response, especially before considering a second dose after relapse.

Disclosure Information N. Klein-González: None. E.A. González-Navarro: None. A. Bartoló-Ibars: None. V. Ortiz-Maldonado: None. M. Torrebadell: None. M. Castellà: None. D. Benítez: None. M. Caballero-Baños: None. R. Cabezón: None. M. Español: None. T. Baumann: None. E. Giné: None. P. Castro: None. J. Esteve: None. J. Yagüe: None. S. Rives: None. Á. Álvaro Urbano-Ispizua: None. J. Delgado: None. M. Juan: None.

\section{P09.06 'AN ENHANCED CRISPR TOOL FOR TREATING CHRONIC MYELOGENOUS LEUKEMIA'}

${ }^{1} \mathrm{D}$ Lainšček ${ }^{*},{ }^{1} \mathrm{~V}$ Forstnerič, ${ }^{1} \mathrm{~S}$ Malenšek, ${ }^{1} \mathrm{M}$ Skrbinek, ${ }^{2} \mathrm{M}$ Sever, ${ }^{1,3} \mathrm{R}$ Jerala. ${ }^{1}$ National institute of Chemistry, Ljubljana, Slovenia; ${ }^{2}$ University medical centre Ljubljana, Ljubljana, Slovenia; ${ }^{3}$ EN-FIST Centre of Excellence, Ljubljana, Slovenia

\subsection{6/jitc-2020-ITOC7.106}

Background Chronic myeloid leukemia (CML) is a myeloproliferative neoplastic disease, occurring in 1 to 2 cases per 100.000 adults, which accounts this type of cancer for approximately $15 \%$ of newly diagnosed leukemia in adult patients. The diagnosis is based upon the genetic translocation between the $\mathrm{t}(9 ; 22)(\mathrm{q} 34 ; \mathrm{q} 11.2)$, resulting in formation 
of Philadelphia fusion chromosome, coding for BCR-ABL1 oncoprotein. The life-long treatment relies on using tyrosine kinase inhibitors (TKIs). In some cases, patients develop point mutations, leading to resistance to TKIs treatment, nearly in $2 \%$. Allogeneic stem cell transplantation is the possible solution for these individuals in late stages of CML with success cure rate only approximately at $40 \% .^{1}$ Based on this funding new solutions for treating cancer with genetic etiology are considered. CRISPR/Cas system, composed of guide RNA, targeting endonuclease Cas9 to specific target genomic region has been used before to mediat breakage of Philadelphia chromosome at the site of oncogenic translocation, although at lower efficiency. ${ }^{2}$

Materials and Methods K562 cells, model for Philadelphia chromosome positive cells, were used. Constructs, expressing BCR-ABL1 targeting gRNA and Cas9, tethered via coiled-coil forming peptides to E.coli exonuclease EXOIII, were nucleofected into target cells. T7E1 assay to detect genome modifications was carried out. TUNEL assay, FACS analysis and bioluminescence measurement were used for cell death determination. SCID mice were used for a subcutaneous K562 cancer model.

Results Our strategy was to couple Cas9 to the exonuclease to promote large deletion at the target site. Of the different exonucleases tested, the EXOIII exhibited the best performance in terms of deletion formation. To improve the rate of deletion genetic lesions, we connected Cas9 and EXOIII via coiled-coil forming peptides, bringing the two enzymes into close proximity (CRISPR-EXO). This resulted in an increased deletion formation compared to the standard CRISPR/Cas system. We performed a case study for the use of the CRISPR-EXO system as a potential anti-cancer therapeutic tool. In the case of our new system, we showed significant increase in cell death due to higher genome modification in BCR-ABL1 region. Later, these findings were confirmed also in an animal cancer model, where animals with tumors, electroporated with CRISPR-EXO system showed full survival and drastic reduction in tumor size.

Conclusions CRISPR-EXO upgraded CRISPR system based on tethering Cas9 protein to exonuclease EXOIII by heterodimeric coiled-coil forming peptides, resulted in highly efficient editing of BCR-ABL1 fusion gene, leading to enhanced death of CML cancer cells.

\section{REFERENCES}

1. Jabbour $\mathrm{E}$, Kantariian H. Chronic myeloid leukemia: 2018 update on diagnosis, therapy and monitoring. Am J Hematol 2018; 93: 442-459.

2. Lekometsev S, Aligianni $\mathrm{S}$, Lapao A, Bürckstümmer T. Efficient generation and reversion of chromosomal translocations using CRISPR/Cas technology. BMC Genomics 2016; 17: 739-745.

Disclosure Information D. Lainšček: None. V. Forstnerič: None. Š. Malenšek: None. M. Skrbinek: None. M. Sever: None. R. Jerala: None.

\section{P09.07 AN IMMUNE MODULATORY VACCINE TARGETING CCL22 PROMOTES ANTI-TUMOR IMMUNITY}

${ }^{1} \mathrm{~L}$ Lecoq ${ }^{*},{ }^{1} \mathrm{KL}$ Kopp, ${ }^{1} \mathrm{R}$ Christensen, ${ }^{1,2} \mathrm{E}$ Martinenaite, ${ }^{1} \mathrm{AW}$ Pedersen, ${ }^{1,2} \mathrm{MH}$ Andersen. ${ }^{1} / O$ Biotech, København, Denmark; ${ }^{2}$ Centre for Cancer Immunotherapy, København, Denmark

10.1136/jitc-2020-ITOC7.107
Background CCL22 is a macrophage-derived chemokine that exerts immunosuppressive functions by the recruitment of regulatory $\mathrm{T}$ cells (Treg) through the CCL22/CCR4 axis. It has been described to play a key role in the suppression of anticancer immunity in different cancer types including ovarian, breast, or pancreatic cancer and is thought to promote the suppression of anti-cancer immunity by Treg recruitment. Recently, we described that CCL22-specific T cells generated from cancer patients can kill CCL22-expressing tumor cells and directly influence the level of CCL2 2 in vitro. ${ }^{1}$ In this study, we provide PoC data for a CCL22-targeting vaccine by assessing the immunotherapeutic efficacy of this approach in syngeneic mouse tumor models.

Materials and Methods Peptide vaccines that induce expansion of CCL22-specific T cells were identified by measurement of vaccine-induced ex vivo response (IFN $\gamma$ ELISpot) in $\mathrm{BALB} / \mathrm{c}$ and $\mathrm{C} 57 \mathrm{BL} / 6$ mice. The antitumor efficacy was evaluated in CT26, Pan02 and B16 syngeneic models. To investigate the vaccine's mode of action, the tumor immune infiltration was analyzed through flow cytometry and qPCR.

Results Vaccination with CCL22-specific peptide vaccines induced expansion of primarily CD8+, CCL22-specific T cell responses (assessed by ex vivo IFN $\gamma$ ELISpot). Treatment with CCL22 vaccines reduced tumor growth and increased survival in CT26, Pan02 and B16 tumor models. Assessment of gene expression in the tumors indicated that vaccination leads to a reduction of CCL22 expression in the tumor microenvironment (TME), as well as the expression of other immune-suppressive molecules such as IDO. Furthermore, vaccinated mice harbored an increased CD $8+\mathrm{T}$ cell infiltration with a concomitant increase in M1/M2 ratio within the TME.

Conclusions This study provides evidence that targeting CCL22 expressing cells by vaccination induces immune modulation in the TME, leading to augmentation of anti-tumor responses - thus provides a rationale for a novel immunotherapeutic approach in cancer.

Disclosure Information I. Lecoq: A. Employment (full or parttime); Modest; IO Biotech. K.L. Kopp: A. Employment (full or part-time); Modest; IO Biotech. R. Christensen: A. Employment (full or part-time); Modest; IO Biotech. E. Martinenaite: A. Employment (full or part-time); Modest; IO Biotech. A.W. Pedersen: A. Employment (full or part-time); Modest; IO Biotech. M.H. Andersen: A. Employment (full or part-time); Modest; IO Biotech.

\section{P09.08 CLINICAL-GRADE MANUFACTURING OF ROR1 CAR T CELLS USING A NOVEL VIRUS-FREE PROTOCOL}

${ }^{1} \mathrm{~K}$ Mestermann*, ${ }^{2} \mathrm{M}$ Eichler, ${ }^{1} \mathrm{M}$ Machwirth, ${ }^{2} \mathrm{~K}$ Kebbel, ${ }^{2} \mathrm{U}$ Köhl, ${ }^{1} \mathrm{H}$ Einsele, ${ }^{2} \mathrm{C}$ Müller,
${ }^{2} \mathrm{~J}$ Lehmann, ${ }^{3 \mathrm{~T}}$ Raskó, ${ }^{3} \mathrm{~F}$ Lundberg, ${ }^{3} \mathrm{Z}$ Izsvák, ${ }^{2} \mathrm{G}$ Schmiedeknecht, ${ }^{1} \mathrm{M}$ Hudecek.
${ }^{1}$ Universitätsklinikum Würrzburg, Würzburg, Germany; ${ }^{2}$ Fraunhofer-Institut für Zelltherapie
und Immunologie, Leipzig, Germany; ${ }^{3}$ Max-Delbrück-Centrum für Molekulare Medizin,
Berlin, Germany
10.1136/jitc-2020-ITOC7.108

Background Immunotherapy with $\mathrm{T}$ cells that were modified by gene-transfer to express a ROR1-specific chimeric antigen receptor (ROR1 CAR-T) has therapeutic potential in ROR1 ${ }^{+}$ malignancies in hematology and oncology. The ROR1 tumor 